Solid pseudopapillary tumor of the pancreas is a rare pancreatic neoplasm that typically occurs in young females. As a low-grade malignancy, the tumor seldom recurs and metastasizes. Management of liver metastasis from such a tumor remains unclear. We present a case of a young girl with liver metastasis due to solid pseudopapillary tumor of the pancreas. She was admitted with chief complaints of epigastric mass on palpation for 16 months and upper abdominal pain for 2 months. An abdominal CT scan detected a mass in the pancreas and multiple nodules in the liver. Then, percutaneous CT-guided tru-cut biopsy was performed and pathological diagnosis of the biopsy material revealed solid pseudopapillary tumor of the pancreas. At 3 months after surgical resection of the pancreatic and liver tumors, CT scan found nodules in her liver. The patient received treatment with transcatheter arterial chemoembolization. She remained well during the 3 years of follow-up with no evidence of recurrence. Therefore, it is suggested that although solid pseudopapillary tumor of the pancreas may be associated with malignant potentiality, a favorable prognosis can also be obtained via rigorous treatment.

Key words: solid pseudopapillary tumor, pancreas, liver metastasis, transcatheter arterial chemoembolization.

Contemp Oncol (Pozn) 2013; 17 (4): 400-403 DOl: $10.5114 /$ wo.2013.37225

\section{Successful treatment of liver metastasis from solid pseudopapillary tumor of the pancreas: a case report}

\author{
Taiguo Liu', Jianping $\mathrm{He}^{1}$, Dan $\mathrm{CaO}^{2}$, Ying Huang ${ }^{3}$
}

1Department of Medical Oncology, Zhejiang Provincial People's Hospital, Hangzhou, China

2Department of Abdominal Cancer, Cancer Center of West China Hospital,

Sichuan University, Chengdu, Sichuan, China

${ }^{3}$ Department of Pathophysiology, West China School of Preclinical and Forensic

Medicine, Sichuan University, Chengdu, Sichuan, China

\section{Introduction}

Solid pseudopapillary tumor (SPT) of the pancreas is a rare pancreatic neoplasm with uncertain etiology that usually occurs in young females. Since Frantz first described SPT in 1959, the number of reported cases has increased [1]. There have been several synonyms for SPT in the literature, such as Franz's tumor, solid and cystic tumor, solid and papillary epithelial neoplasm, papillary-cystic neoplasm, papillary cystic epithelial neoplasm and papillary-cystic tumor. In 1996, the World Health Organization (WHO) renamed this tumor as SPT in the International Histological Classification of Tumors [2]. Solid pseudopapillary tumor is generally considered to have a low potential for malignancy, which is often localized in the pancreas and is rarely a metastatic disease. Because of its rareness and unusual behavior, SPT is often associated with diagnostic and therapeutic challenges. Surgical resection is now considered the most efficient treatment option for patients with SPT, because it offers a good chance of long-term survival. However, there is a lack of data on the management of liver metastasis in patients with SPT. We report a case of a patient with pancreatic SPT with liver metastasis, who obtained a favorable outcome after rigorous treatment.

\section{Case report}

A 19-year-old female was admitted to our hospital in January 2009 with chief complaints of epigastric mass on palpation for 16 months and upper abdominal pain for 2 months. She had lost 10 kilograms of weight in a single month. Her past medical history was unremarkable. Physical examination revealed a hard mass in the upper abdomen. A $13 \mathrm{~cm}$ cystic lesion of the pancreas was detected by abdominal ultrasonography. Findings of routine laboratory tests, including carcinoembryonic antigen (CEA) and cancer antigen 19-9 (CA 19-9), were within the normal range. An abdominal CT scan found a giant solid mass $(14.8 \mathrm{~cm} \times 8.9 \mathrm{~cm})$ in the pancreatic body, multiple nodules in the anterior and posterior segment of the right lobe of the liver about $8.5 \mathrm{~cm} \times 3.7 \mathrm{~cm}$ and $7.2 \mathrm{~cm} \times 3.1 \mathrm{~cm}$ and splenomegaly (Fig. 1). Subsequently, we performed percutaneous CT-guided tru-cut biopsy of the tumor and pathological diagnosis of the biopsy material revealed pancreatic SPT.

Given the patient's young age as well as the location of the pancreatic tumor, which was not situated in the tail but in the body, pancreaticoduodenectomy apart from distal pancreatectomy, hepatic tumor resection and splenectomy was conducted. A large mass in the pancreas with multiple metastatic nodules in the liver was seen during the operation. Her post-operative course was unremarkable, and she was discharged 1 week after her surgery with routine follow-ups. 

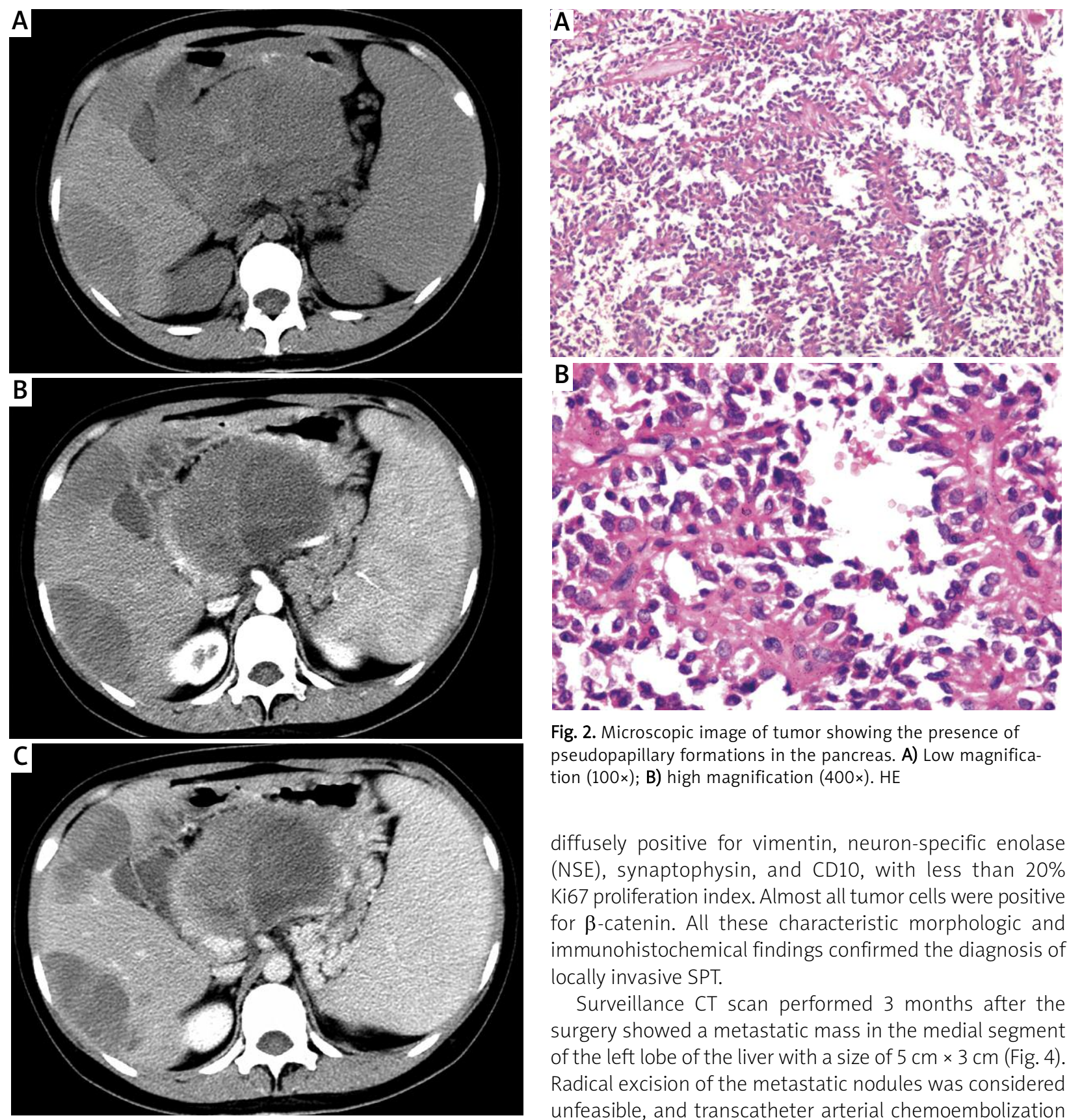

Fig. 2. Microscopic image of tumor showing the presence of pseudopapillary formations in the pancreas. A) Low magnification (100x); B) high magnification (400x). HE

diffusely positive for vimentin, neuron-specific enolase (NSE), synaptophysin, and CD10, with less than $20 \%$ Ki67 proliferation index. Almost all tumor cells were positive for $\beta$-catenin. All these characteristic morphologic and immunohistochemical findings confirmed the diagnosis of locally invasive SPT.

Surveillance CT scan performed 3 months after the surgery showed a metastatic mass in the medial segment of the left lobe of the liver with a size of $5 \mathrm{~cm} \times 3 \mathrm{~cm}$ (Fig. 4). Radical excision of the metastatic nodules was considered unfeasible, and transcatheter arterial chemoembolization

Fig. 1. Abdominal CT scan on admission revealed a giant solid mass $(14.8 \mathrm{~cm} \times 8.9 \mathrm{~cm})$ in the pancreatic body, multiple nodules in the anterior and posterior segment of right lobe of liver about $8.5 \mathrm{~cm} \times 3.7 \mathrm{~cm}$ and $7.2 \mathrm{~cm} \times 3.1 \mathrm{~cm}$ and splenomegaly.

A) Unenhanced CT; B) early phase of contrast-enhanced $C T$; C) portal phase of contrast-enhanced CT

The resected pancreatic lesion measured about $14.8 \mathrm{~cm}$ $\times 10 \mathrm{~cm} \times 8.9 \mathrm{~cm}$. When dissecting the mass, we found a heterogeneous solid appearance with areas of hemorrhage and extensive necrosis. No invasion into the spleen was identified. Microscopically, solid areas mainly consisting of monomorphic epithelioid cells and a moderate cellular atypia without mitotic activity were found (Fig. 2). Characteristic pseudopapillary formations were frequently observed in the liver metastases (Fig. 3). Immunohistochemical studies of the primary lesion revealed that the tumor cells were (TACE) with epirubicin and iodized oil was performed 3 times in total. Adjuvant chemotherapy or radiotherapy were not offered, because very few reports regarding the use of such therapies for pancreatic SPT existed with only limited response $[3,4]$. And then, follow-up abdominal and chest CT scans were done every 3 months in the first half year and every 6 months afterwards, and no tumor in the pancreas or in the liver was detected. The patient remained diseasefree at 35 months after TACE, i.e. 38 months after removal of the primary pancreatic tumor.

\section{Discussion}

Solid pseudopapillary tumor is a rare and indolent neoplasm of the pancreas with a favorable prognosis after resection. It accounts for only approximately 1 percent of all primary pancreatic tumors [5]. However, several studies have 

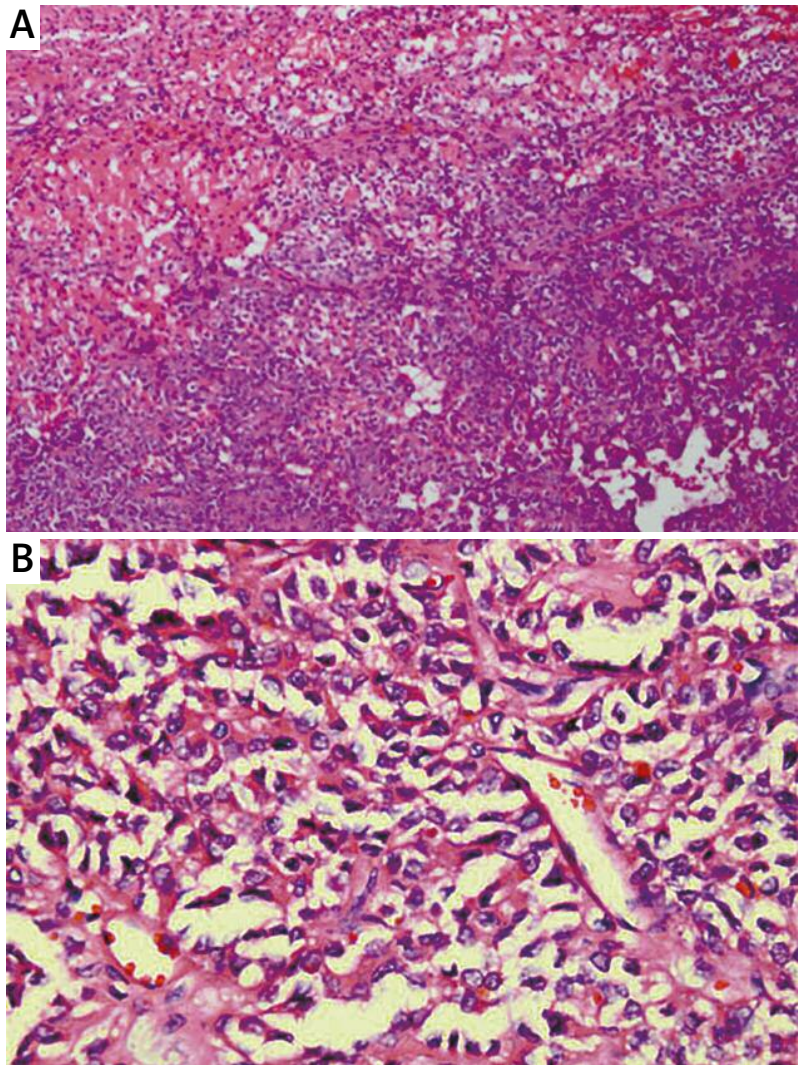

Fig. 3. Microscopic image of tumor showing the presence of pseudopapillary formations in the liver metastases.

A) Low magnification (100x); B) high magnification (400x). HE

indicated that the incidence of SPT is increasing. It usually occurs in young women in the second or third decade of life, with an average female: male ratio of $5: 1.9$ [6], which is up to $10: 1$ in certain studies [7].

The tendency of SPN to afflict predominantly young women leads to the hypothesis that its pathogenesis may be influenced by sex hormones. Yeh et al. have reported that the progesterone receptor is uniquely expressed in SPT while both estrogen and progesterone receptors are expressed in mucinous cystic neoplasm [8]. Tien et al. reported no significant differences in qualitative sex hormone receptor status between male and female SPT patients [9]. Clinical studies have shown that the progesterone receptor is expressed in all cases of SPT. And in our report, the patient tested negative for both estrogen and progesterone receptors. Thus, more evidence has to be collected to clarify the potential impact of sex hormones on the pathogenesis of SPT.

The origin of SPT still remains elusive. Immunohistochemical stains show a variety of expressions, such as epithelial, neural and stromal elements. A previous study hypothesized that it might be a tumor derived from the pancreatic duct cells, acinar cells, and endocrine cells [10]. Recent evidence indicates that SPT may arise from the centroacinar cell of the pancreas [11]. Based on both immunohistochemical staining and electron microscopic findings, a potential link between tumor cells and centroacinar cells is identified. However, further studies are still needed to confirm this correlation.


Fig. 4. Three months after the surgery, CT revealed a metastatic mass in the medial segment of the left lobe of liver with a size of $5 \mathrm{~cm} \times 3 \mathrm{~cm}$. A) Unenhanced CT; B) early phase of contrastenhanced $\mathrm{CT} ; \mathrm{C}$ ) portal phase of contrast-enhanced $\mathrm{CT}$

Like solid variant of serous cystadenoma of the pancreas, a rare tumor of the pancreas, there often has been a delay in diagnosis until the discovery of a large tumor mass during abdominal examination or imaging [12]. The prognosis of SPT is favorable because of its low malignant potential. It appears to be capable of local invasion but usually does not metastasize to distant sites. Some studies observed that metastases or tumor recurrence were detected in about 15\% of cases of SPT [13]. The site most frequently affected by metastases is the liver. Other sites that may have metastatic masses include the vena cava, spleen, peritoneum and duodenum [14]. In our report, ultrasonography and CT revealed a large mass in the pancreatic body and multiple nodules in the liver when the patient was admitted to the hospital. After complete resection of these tumors, liver 
metastases appeared again 3 months following the surgery, indicating malignancy of the tumor.

Generally speaking, surgery is the best choice of treatment even in cases of distant metastasis, because other adjuvant therapies have not shown demonstrable responses. Local resection or enucleation can be performed when the tumor is small. Distal pancreatectomy combined with or without splenectomy can be performed for pancreatic body and/or tail tumor, and pancreatoduodenectomy for pancreatic head tumor. Considering the multiple liver metastases in this case, we performed aggressive surgical resection to remove SPT, spleen and liver nodules. However, at 3 months after the surgery, liver metastases recurred. The patient received TACE treatment and she remained disease-free at 35 months after TACE. Follow-up CT scans did not detect any nodes in the liver after TACE.

Our report suggests that although pancreatic SPT may be associated with malignant potentiality, a favorable prognosis can also be obtained via rigorous treatment. TACE with Pharmorubicin and iodized oil is an effective treatment option for the control of liver metastasis of SPT.

The authors declare no conflict of interest.

\section{References}

1. Lin MY, Stabile BE. Solid pseudopapillary neoplasm of the pancreas: a rare and atypically aggressive disease among male patients. Am Surg 2010; 76: 1075-8.

2. Kloppel G, Solcia E, Longnecker DS, Capella C, Sobin LH. Histolog ical typing of tumors of the exocrine pancreas. 2nd ed. SpringerVerlag; Berlin 1996.

3. Lam KY, Lo CY, Fan ST. Pancreatic solid-cystic-papillary tumor: clinicopathologic features in eight patients from Hong Kong and review of the literature. World J Surg 1999; 23: 1045-50.

4. Fried P, Cooper J, Balthazar E, Fazzini E, Newall J. A role for radiotherapy in the treatment of solid and papillary neoplasms of the pancreas. Cancer 1985, 56: 2783-5.

5. Klimstra DS, Wenig BM, Heffess CS. Solid-pseudopapillary tumor of the pancreas: a typically cystic carcinoma of low malignant potential. Semin Diagn Pathol 2000; 17: 66-80.

6. Choi JY, Kim MJ, Kim JH, et al. Solid pseudopapillary tumor of the pancreas: typical and atypical manifestations. AJR Am J Roentgenol 2006; 187: W178-86.

7. Mulkeen AL, Yoo PS, Cha C. Less common neoplasms of the pancreas. World J Gastroenterol 2006; 12: 3180-5.

8. Yeh TS, Jan YY, Chiu CT, et al. Characterisation of oestrogen receptor, progesterone receptor, trefoil factor 1 , and epidermal growth factor and its receptor in pancreatic cystic neoplasms and pancreatic ductal adenocarcinoma. Gut 2002; 51: 712-6.

9. Tien YW, Ser KH, Hu RH, Lee CY, Jeng YM, Lee PH. Solid pseudopapillary neoplasms of the pancreas: is there a pathologic basis for the observed gender differences in incidence? Surgery 2005;137: 591-6.

10. Coleman KM, Doherty MC, Bigler SA. Solid-pseudopapillary tumor of the pancreas. Radiographics 2003; 23: 1644-8.

11. Kallichanda N, Tsai S, Stabile BE, Buslon V, Delgado DL, French SW. Histogenesis of solid pseudopapillary tumor of the pancreas: the case for the centroacinar cell of origin. Exp Mol Pathol 2006; 81: 101-7.

12. Yasuda A, Sawai H, Ochi N, Matsuo Y, Okada Y, Takeyama H. Solid variant of serous cystadenoma of the pancreas. Arch Med Sci 2011; 7: 353-5.

13. Martin RC, Klimstra DS, Brennan MF, Conlon KC. Solid-pseudopapillary tumor of the pancreas: a surgical enigma? Ann Surg Oncol 2002 9: 35-40.
14. Camacho-Aguilera JF, Romero-Mejía C, Valenzuela-Espinoza A. Solid pseudopapillary tumor of the pancreas: case report and literature review. Cir Cir 2010; 78: 73-8.

\section{Address for correspondence}

\section{Taiguo Liu PhD}

Department of Medical Oncology

Zhejiang Provincial People's Hospital

No. 158, Shangtang Road Hangzhou,

Zhejiang Province 310014 Hangzhou, China

e-mail: liutaiguo031@126.com

Submitted: 13.03 .2012

Accepted: $\quad$ 20.09.2012 